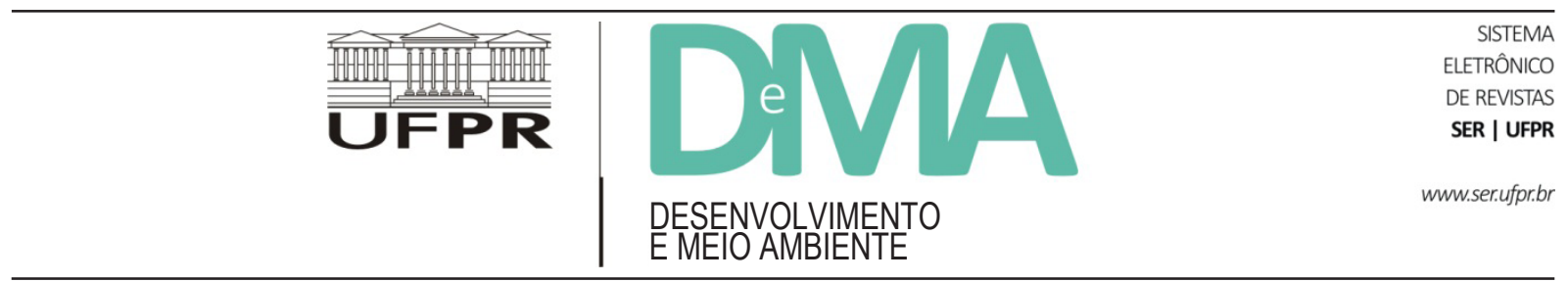

\title{
Examinando as contradições em torno das Pequenas Centrais Hidrelétricas como fontes sustentáveis de energia no Brasil
}

\section{Examining the Contradictions of Small Hydro power Plants as Sustainable Energy Sources in Brazil}

\author{
Juliana Ribeiro LATINI ${ }^{1 *}$, Marcos Antonio PEDLOWSKI ${ }^{1}$ \\ ${ }^{1}$ Universidade Estadual do Norte Fluminense Darcy Ribeiro (UENF), Campos dos Goytacazes, RJ, Brasil. \\ *E-mail de contato: jrlbio@gmail.com
}

Artigo recebido em 12 de agosto de 2015, versão final aceita em 20 de abril de 2016.

RESUMO: $\quad$ As Pequenas Centrais Hidrelétricas (PCHs) vêm ganhando destaque nos esforços voltados para a expansão da oferta de energia elétrica em âmbito mundial. Tal fato se deve à ideia de que essas plantas de geração de energia, por seu menor porte, causariam impactos ambientais insignificantes, quando comparadas com grandes usinas hidrelétricas. Em função disso, o Brasil tem flexibilizado as normas ambientais e concedido incentivos financeiros com o objetivo de facilitar e agilizar a implantação de PCHs no país. Além disso, programas governamentais, como o PROINFA e o "Luz para Todos", também podem ter contribuído para a expansão desse tipo de empreendimento nos últimos anos. Apesar da imagem limpa e dos incentivos concedidos, diferentes pesquisas científicas têm demonstrado evidências de que as PCHs, mesmo com seu menor porte, alteram as características hidrológicas dos ecossistemas aquáticos e causam impactos à biota em níveis individual, populacional e de comunidades. Em adição, já existem evidências de que alguns impactos ambientais acarretados por um conjunto de PCHs podem ser maiores do que os impactos causados por usinas hidrelétricas de grande porte. Portanto, não existem motivos para facilitar e agilizar os processos de aprovação e implementação de PCHs. O presente artigo aponta que as estratégias utilizadas para acelerar a implantação de PCHs contribuem para fragilizar os instrumentos de controle ambiental adotados no Brasil e para disseminar a percepção de que esses instrumentos são entraves ao desenvolvimento econômico, colocando, assim, a questão ambiental em patamares inferiores nas decisões tomadas no setor elétrico. $\mathrm{O}$ artigo postula, ainda, que as $\mathrm{PCHs}$, como qualquer outro empreendimento potencialmente poluidor, devem ser alvo de uma avaliação socioambiental criteriosa, que possa subsidiar o processo decisório sobre as alternativas para a expansão da oferta de energia elétrica no Brasil.

Palavras-chave: $\mathrm{PCH}$; impactos ambientais; incentivos governamentais. 
ABSTRACT: Small Hydropower Plants (SHPs) have become prominent worldwide because of widespread efforts to expand energy supply. This development is partially due to the misguided idea that this type of power generation plants can cause insignificant environmental impacts when compared to their larger counterparts. As a result of this point of view, Brazil has relaxed environmental standards and granted financial incentives to facilitate and expedite the implementation of SHP. In addition, government programs such as the PROINFA and "Luz para todos" also contributed to the expansion of SHPs. Despite of the clean image and granted incentives to SHPs, there is scientific evidence to support the claim that SHPs, despite its smaller size, can alter the hydrological characteristics of aquatic ecosystems with impacts to biota at individual, population and community levels. In addition, there is scientific evidence showing that the environmental impacts caused by a number of SHPs may be larger than the impacts caused by large hydroelectric plants. Therefore, we argue that there is no reason to facilitate and streamline the procedures for the approval and implementation of SHPs. This article discusses the strategies used to accelerate the deployment of SHPs which weaken the environmental control instruments adopted in Brazil. We also address the perception that the environmental licensing instruments are obstacles to economic development. We argue that this type of behavior contributes to halt the position of the environmental dimension in the decision-making process by the electric energy sector. Finally, we proposed that SHPs, as any other potentially polluting development, should be subject to careful environmental assessments which can support the decision-making process regarding the establishing of sustainable alternatives for the expansion of electricity supply in Brazil.

Keywords: SHP; environmental impacts; governmental incentives.

\section{Introdução}

Os corpos d'água naturais vêm sendo modificados pela construção de barragens com o objetivo de atender as demandas dos seres humanos para diversas finalidades, tais como irrigação, abastecimento público e produção de energia (Mello \& Piasetin, 2011). Durante o século XX, grandes barragens foram consideradas as melhores alternativas para atender as necessidades humanas, tendo sido vistas como sinônimo de desenvolvimento e crescimento econômico (WCD, 2000).

No que diz respeito à produção de energia, grandes barragens são construídas para a implantação de usinas hidrelétricas, as quais foram associadas por muito tempo à imagem de fonte limpa e renovável de energia (Abassi \& Abassi, 2011). Entretanto, mais recentemente, essa imagem sustentável vem sendo questionada em função da demonstração de que grandes impactos ambientais e sociais são causados pela implantação deste tipo de empreendimento, o que tem contribuído para uma diminuição do apoio aos mesmos (Bermann, 2007; Abassi \& Abassi, 2011; Premalatha et al., 2014). Em função desse processo de descrédito das grandes centrais hidrelétricas, as chamadas Pequenas Centrais Hidrelétricas (PCHs) vêm ganhando espaço como uma alternativa mais sustentável e vêm sendo adotadas por diversos países, como China, Índia, Canadá, Reino Unido e Brasil (Abassi \& Abassi, 2011).

As PCHs são plantas de produção de energia elétrica com menor capacidade instalada em megawatts (MW), mas ainda não existe um consenso mundial sobre o valor máximo de capacidade instalada desse tipo de empreendimento (IPCC, 2012). Apesar da pequena capacidade instalada, as PCHs ainda requerem a construção de uma barragem, seja para direcionar o fluxo d'água ou para a criação de um reservatório para fornecer a vazão necessária ao funcionamento da $\mathrm{PCH}$ (Eletrobrás, 2000). No Brasil, são considerados PCHs 
os aproveitamentos hidrelétricos com potência entre 1 e $30 \mathrm{MW}$, que são destinados à produção independente, autoprodução ou produção independente autônoma, com áreas de reservatório menores do que $3,0 \mathrm{~km}^{2}$, havendo certa flexibilização quanto à dimensão do mesmo (ANEEL, 2003a).

De acordo com o Banco de Informações de Geração ${ }^{1}$ (BIG), da ANEEL, estão instaladas 473 PCHs em todo o território brasileiro, com uma capacidade instalada de aproximadamente 4.800 MW. Com base ainda nos dados do BIG, $168 \mathrm{PCHs}$ já possuem potência outorgada, o que deverá gerar 2.332,67 MW de potência adicional à capacidade elétrica instalada no Brasil. Esses números podem ainda aumentar, pois, de acordo com Tiago et al. (2011), o potencial hidroenergético para PCHs disponível no Brasil é de $15.500 \mathrm{MW}$, o que, considerando que a potência média de uma PCH é de $10 \mathrm{MW}$, permitiria a instalação de mais 1.550 novas PCHs no país.

Atualmente, as PCHs fazem parte das fontes alternativas de energia renovável, juntamente com as fontes de energia eólica, fotovoltaica e a partir de biomassa (EPE, 2014). As fontes alternativas contribuem para a diversificação da matriz elétrica e para a segurança energética do país, além de solucionarem as demandas de energia elétrica locais, por configurarem plantas de geração distribuída (Lopes, 2015). Nesse contexto, as PCHs possuem vantagens por serem adequadas para aproveitar o grande potencial hidroenergético do Brasil, sendo adequadas para rios de menores dimensões em locais isolados e por terem disponível tecnologia conhecida e nacional para sua implantação, gerando energia a preços competitivos (Castro et al., 2009). Outra vantagem das PCHs é que elas são adequadas para substituir pequenas termelétricas, que historicamente atendem as demandas de energia dos sistemas elétricos isolados, contribuindo para a redução da emissão de gases do efeito estufa e para o combate ao aquecimento global (Marrocos Neto \& Moret, 2008).

No entanto, é importante ressaltar que, apesar do grande potencial existente no Brasil e da imagem de fonte de energia de baixo impacto ambiental atribuída às PCHs, a opção por esse tipo de empreendimento para a expansão da oferta de energia elétrica deveria ser analisada mais cuidadosamente. $O$ fato é que diferentes pesquisadores têm questionado a falta de evidências científicas de que as PCHs causam menores impactos ambientais por unidade de energia gerada do que as grandes usinas hidrelétricas, o que torna a imagem "ecoamigável" das PCHs discutível (Abassi \& Abassi, 2000; 2011; Premalatha et al., 2014).

Neste artigo visa-se realizar um levantamento dos aspectos que representam incentivos à implantação de PCHs no Brasil. Complementarmente, apresenta-se uma avaliação dos impactos advindos da disseminação desses empreendimentos, tendo como base uma revisão dos impactos já identificados por outros pesquisadores em níveis nacional e internacional. Pretende-se, desta forma, contribuir para o debate em curso acerca da sustentabilidade das estratégias que estão sendo adotadas para expandir a capacidade de geração de energia elétrica no Brasil.

\section{Fatores que favorecem a implantação de PCHs no Brasil}

As PCHs passaram a ser alvo do planejamento elétrico brasileiro na década de 1990, como resultado de uma combinação entre as novas demandas 
energéticas que surgiam no país após a implantação do Plano Real e a decisão de afastar o Estado da intervenção direta na construção deste tipo de empreendimento. Essa conjuntura impulsionou a abertura do setor elétrico ao investimento privado, para que novas unidades geradoras de eletricidade fossem implementadas de forma rápida, fazendo, assim, com que as PCHs ganhassem proeminência no cenário nacional (Souza et al., 2002).

Além do contexto observado na década de 1990, uma hipótese que pode ser levantada sobre o aumento do interesse pela implantação de PCHs no Brasil é a de que essas plantas de geração são mais adequadas do que as grandes usinas para aproveitar os potenciais hidrelétricos remanescentes nas regiões não amazônicas do país. De acordo com Moretto et al. (2012), atualmente, os potenciais hidrelétricos apropriados para a implantação de grandes usinas hidrelétricas só se encontram disponíveis na região amazônica, pois são escassos nas outras regiões. Isso torna as outras regiões brasileiras alvos de interesse para o aumento da oferta de energia por meio de PCHs, visto que os potenciais ainda disponíveis não comportam a instalação de grandes usinas. Essa tendência pode ser observada na Figura 1, que ilustra uma preferência pela instalação de $\mathrm{PCHs}$ nas regiões Sul, Sudeste e Centro-Oeste do país.

Diante desse contexto, que reúne disponibilidade de potencial hidrelétrico para empreendimentos de menor capacidade instalada e aumento da demanda nacional por energia elétrica, diversas estratégias podem ser identificadas como fatores com potencial para aumentar a atratividade das PCHs e estimular a implantação e a disseminação desse tipo de empreendimento pelo Brasil.
A primeira estratégia que pode ser destacada diz respeito à alteração dos critérios para enquadramento de um aproveitamento hidrelétrico como $\mathrm{PCH}$. A primeira regulamentação a tratar do assunto, a Portaria DNAEE ${ }^{2} 109$, de 1982, trazia uma série de restrições de engenharia e o limite máximo de capacidade instalada de $10 \mathrm{MW}$. Nas regulamentações subsequentes (Portaria DNAEE 136, de 1987, e Resolução ANEEL 33 394, de 1998), as restrições de engenharia foram extintas, a capacidade máxima instalada foi ampliada para $30 \mathrm{MW}$ e uma área máxima de $3 \mathrm{~km}^{2}$ para o reservatório foi definida. A Resolução ANEEL 652, de 2003, que está em vigor atualmente, mantém a capacidade máxima em $30 \mathrm{MW}$, inclui critérios de modalidade de exploração do aproveitamento e flexibiliza o tamanho do reservatório, que pode possuir até 13 $\mathrm{km}^{2}$ para fins de aproveitamento exclusivamente hidrelétrico, além de poder ter seu tamanho definido em função de usos múltiplos, sem um limite máximo estabelecido na resolução para esses casos (DNAEE, 1982; 1987; ANEEL, 1998; 2003a).

$\mathrm{Na}$ interpretação de Albuquerque \& Moraes (2013), essas alterações nos critérios de enquadramento de PCHs têm o objetivo de incluir o maior número possível de empreendimentos nessa categoria, facilitando a implantação desse tipo de unidade geradora de energia, legitimada pela crença de que as mesmas produzem energia limpa e com baixos impactos ambientais.

Outro fato que pode ser observado como incentivo à implantação de $\mathrm{PCHs}$ foi a dispensa de licitação para a concessão do direito de explorar o potencial hidrelétrico, bastando apenas autorização da ANEEL, além de benefícios fiscais, financeiros e comerciais, podendo ser citados (EPE, 2009):

\footnotetext{
Departamento Nacional de Águas e Energia Elétrica (órgão extinto). Agência Nacional de Energia Elétrica.
} 


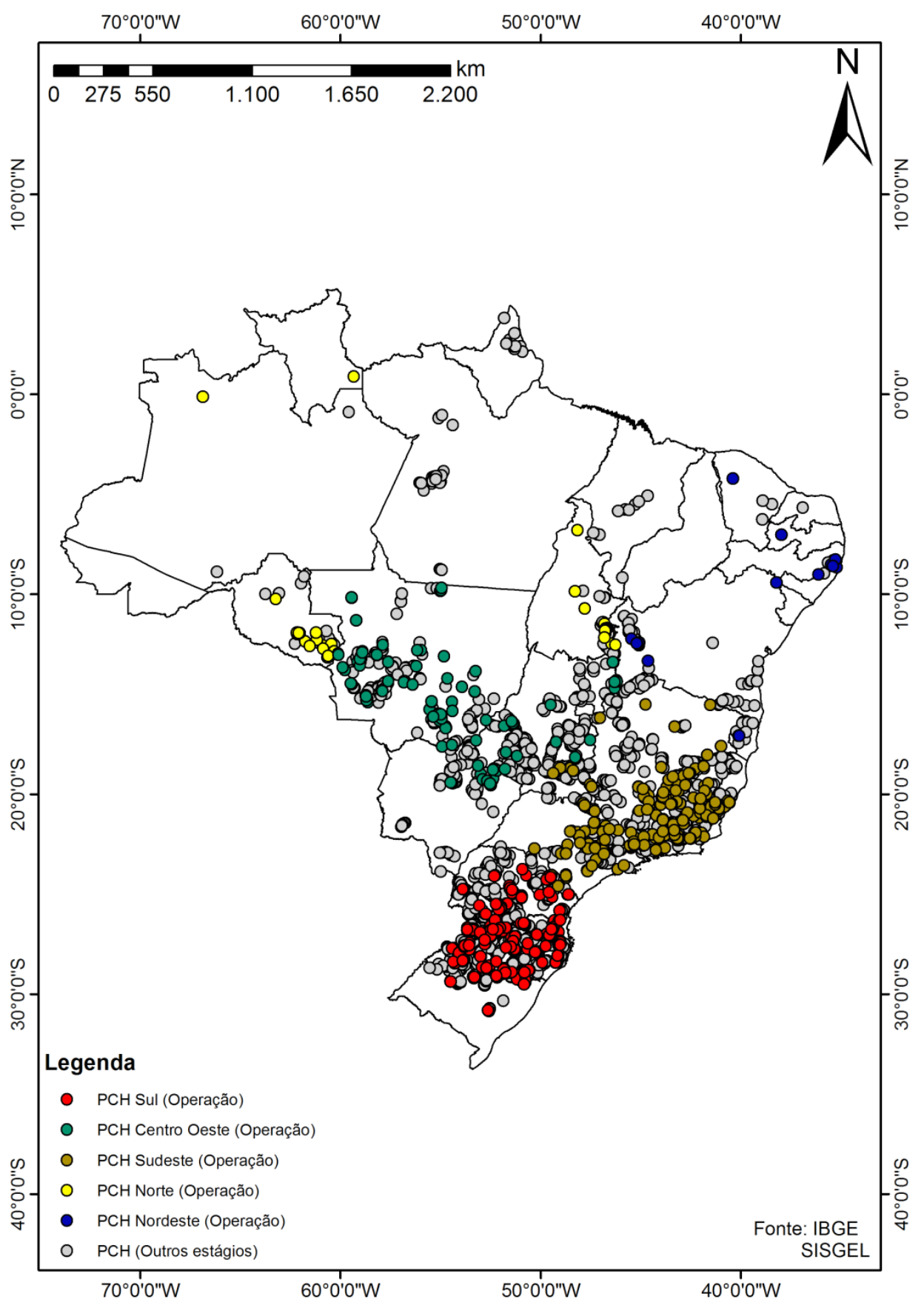

FIGURA 1 - Distribuição espacial das PCHs no Brasil. Elaborado por LCA/UENF a partir de dados do Sisgel/ ANEEL. Dados acessados em agosto de 2015. 
- Isenção da obrigação de aplicar $1 \%$ da receita operacional líquida, anualmente, em pesquisa e desenvolvimento do setor elétrico;

- Isenção da obrigação de pagar compensação financeira aos estados e municípios pelo uso dos recursos hídricos;

- Possibilidade de comercialização diretamente com consumidores cuja carga seja maior ou igual a $500 \mathrm{KW}$;

- Possibilidade de recebimento de subsídio do Fundo Conta de Consumo de Combustíveis Fósseis (CCC) para empreendimentos instalados nos sistemas isolados;

- A redução de no mínimo 50\% dos encargos por uso das redes de transmissão e distribuição.

Além desses benefícios, alguns programas governamentais foram criados para incentivar a ampliação do número de PCHs no país. O Programa de Incentivo às Fontes Alternativas de Energia Elétrica (PROINFA) foi regulamentado em 2004, com o objetivo manifesto de aumentar a participação da energia elétrica produzida com base em fontes eólica, PCHs e biomassa, na matriz energética brasileira. Tal regulamentação garantiu a compra da energia elétrica gerada pelas instalações integrantes do PROINFA, pela Eletrobrás, por um período de 20 anos, a contar da data prevista para a operação do empreendimento (Brasil, 2002; 2004). Essa medida visou tornar o programa não vulnerável às oscilações de mercado, constituindo mais um atrativo ao investimento em PCHs (Leão \& Brasil Junior, 2008). O programa governamental "Luz para Todos", lançado pelo governo federal em 2003, que tinha como objetivo universalizar o fornecimento de energia elétrica, também pode ter favorecido a construção de PCHs. As PCHs são consideradas alternativas eficientes para o cumprimento das me- tas do programa, pois, em função do menor porte, podem ser instaladas em locais isolados e zonas rurais, que eram justamente os alvos preferenciais do programa (Tiago, 2004).

Em adição aos programas nacionais, outro aspecto com potencial para estimular a instalação de PCHs é a possibilidade do registro desses empreendimentos como Mecanismos de Desenvolvimento Limpo (MDL), os quais foram estabelecidos a partir da assinatura do Protocolo de Kyoto. Dessa forma, as $\mathrm{PCHs}$ podem participar do mercado de créditos de carbono, o que permite o aumento da receita produzida, que passa a somar a receita gerada pela venda de energia com a receita gerada pela comercialização dos créditos de carbono (Barbosa \& Souza, 2009), aumentando assim a atratividade econômica dos empreendimentos considerados PCHs.

Ao mesmo tempo em que esses incentivos são conferidos visando aumentar a atratividade das PCHs, facilitações no processo de licenciamento desses projetos têm sido realizadas visando agilizar o procedimento e sustentar essa imagem atrativa. Essas facilitações se deram por meio da Resolução CONAMA 279/2001, que trata do licenciamento ambiental de empreendimentos elétricos classificados como projetos de baixo impacto, incluindo-se aí as PCHs. De acordo com a resolução 279/2001, as PCHs podem ser enquadradas em um licenciamento ambiental simplificado, baseado na apresentação de um Relatório Ambiental Simplificado (RAS) e não mais na apresentação do Estudo de Impacto Ambiental (EIA) e seu respectivo Relatório de Impacto Ambiental (RIMA), como era preconizado pela Resolução CONAMA 001/1986 anteriormente. Outra flexibilização trazida pela Resolução CONAMA 279/2001 foi a alteração dos prazos para a emissão das licenças ambientais. Anteriormente, o prazo dado pela Resolução CONAMA 237/1997 era de 
até seis meses para realizar as análises necessárias e decidir sobre a emissão das licenças. Desde 2001, nos casos de licenciamento ambiental simplificado, o órgão ambiental deve realizar as análises e tomar a decisão sobre o deferimento ou indeferimento das licenças em no máximo 60 dias (CONAMA, 2001), ocasionando, assim, uma redução de dois terços no tempo disponível para a análise dos impactos causados pelo empreendimento.

As alterações no processo de licenciamento ambiental para empreendimentos elétricos com pequeno potencial de impacto foram consequência da crise energética e do "apagão" que ocorreram no setor elétrico em 2001. Essa conjuntura de aumento da demanda possibilitou a fragilização das regulamentações ambientais visando facilitar e agilizar a implantação de empreendimentos elétricos em nome da urgência de se aumentar a oferta de energia elétrica e solucionar a crise energética vivida pelo Brasil naquele momento (Albuquerque \& Moraes, 2013).

Todas as evidências aqui apresentadas mostram que diversos benefícios, incentivos e flexibilizações foram implementados para estimular a implantação de PCHs. O próprio "Guia do empreendedor de pequenas centrais hidrelétricas" (ANEEL, 2003b) reconhece que benefícios foram concedidos e que os procedimentos foram simplificados com objetivo de tornar as $\mathrm{PCHs}$ mais atrativas dos pontos de vista econômico e financeiro, ratificando as informações apresentadas nesta seção.

\section{Impactos ambientais e sociais de PCHs}

A integridade ecológica de um ecossistema aquático está intimamente ligada ao regime de fluxo de água desse ecossistema (Poff et al., 1997). Assim, a construção de uma barragem em um rio, tanto para produção de energia quanto para outros usos, altera drasticamente esse regime, modificando a dinâmica natural desse ecossistema (Baxter, 1977). As modificações causadas pela construção de barragens acarretam alterações de três ordens: (1) das características físicas, químicas e geomorfológicas do rio; (2) na produtividade primária; (3) nas comunidades de consumidores, como consequência dos impactos de primeira e segunda ordem (WCD, 2000).

Os impactos socioambientais das grandes barragens são bem conhecidos. De acordo com Kahn et al. (2014), as grandes barragens construídas para a implantação de usinas hidrelétricas podem acarretar impactos globais, regionais (abrangendo toda a bacia hidrográfica) e locais. De acordo com Kahn e colaboradores, os impactos globais dizem respeito à influência que as grandes barragens podem exercer no clima em função da perda de vegetação pelo enchimento do reservatório e, consequentemente, da alteração das taxas de fotossíntese e decomposição. Os impactos na escala da bacia hidrográfica são resultados da sinergia entre os diferentes impactos de uma única usina hidrelétrica, bem como da sinergia dos impactos desta com os impactos de outros usos da terra preexistentes. Finalmente, Kahn e seus colaboradores apontam que os impactos locais são as consequências ambientais e sociais que ocorrem em torno da barragem e do reservatório da hidrelétrica, causados pelo(a): (1) bloqueio do fluxo de sedimentos; (2) alteração do ciclo de cheia e seca do rio; (3) impedimento da migração de peixes; redução dos níveis de oxigênio à montante e à jusante da barragem; (3) fragmentação do ecossistema aquático; (4) deslocamento de populações; (5) perda de acesso aos serviços ecológicos.

Por outro lado, os impactos causados por pequenas barragens, como as construídas para as PCHs, ainda são pouco documentados, pois se 
assumiu que, mesmo no pior cenário, tais empreendimentos só causariam impactos insignificantes, principalmente em função de seu porte reduzido (Abassi \& Abassi, 2011; Premalatha et al., 2014). Entretanto, diversas pesquisas vêm mostrando uma tendência contrária, indicando que as $\mathrm{PCHs}$, afetam as características físicas dos rios e as comunidades de fauna e flora existentes (Tabela 1). Apesar de a maioria dessas pesquisas terem sido realizadas em âmbito internacional e seus resultados não poderem ser rigorosamente reproduzidos para o Brasil, os mesmos indicam que as $\mathrm{PCHs}$ possuem diferentes impactos sobre o ambiente, mesmo com seu porte reduzido.

Essas pesquisas mostraram que a construção de barragens e o funcionamento das PCHs alteram as características físicas do rio, modificando a frequência de ambientes lênticos e lóticos e alterando, também, a estrutura de habitat existente ao longo do rio (Fu et al., 2008; Zhou et al., 2009; Wu et al., 2009; 2012; Benejan et al., 2014). Essa alte- ração das condições físicas dos ecossistemas pode ocasionar mudanças à biota em níveis individuais, bem como alterar a estrutura de populações e comunidades. Este processo se dá porque as alterações ocorridas podem interferir diretamente nos tipos e na qualidade de habitats existentes, modificando, assim, a compatibilidade dos nichos ecológicos das espécies com as novas características ambientais (Ricklefs, 2010).

No tocante aos impactos das PCHs sobre a ictiofauna, efeitos ecológicos foram apontados em três níveis, por meio da redução das dimensões corporais de peixes de diferentes espécies e de alterações na estrutura de populações e de comunidades (Almodóvar \& Nicola, 1999; Hirschmann et al. 2008; Benejan et al., 2014). As alterações na estrutura de populações foram evidenciadas, no geral, por meio da redução da biomassa, abundância e densidade. Uma pesquisa relatou ainda mudanças na estrutura etária de uma população de peixes, a qual passou a apresentar indivíduos mais velhos

TABELA 1 - Alterações ambientais causadas por PCHs.

\begin{tabular}{ll}
\hline \multicolumn{1}{c}{ Impactos } & \multicolumn{1}{c}{ Referências } \\
\hline $\begin{array}{l}\text { Alteração das características físicas do rio, como velocidade do fluxo, profundidade do } \\
\text { canal e largura do canal, e alteração da disponibilidade de refúgios para a fauna }\end{array}$ & $\begin{array}{l}\text { Fu } \text { et al., 2008; Zhou } \text { et al., 2009; } \\
\text { Wu et al., 2009; 2012; Benejan et } \\
\text { al., 2014 }\end{array}$ \\
$\begin{array}{l}\text { Ictiofauna: redução da densidade populacional, redução da biomassa populacional, } \\
\text { redução da abundância populacional, alteração da abundância relativa, alteração de } \\
\text { ordens dominantes, desaparecimento de espécies raras, alteração da estrutura etária, } \\
\text { redução de peso e comprimento dos indivíduos }\end{array}$ & $\begin{array}{l}\text { Almodóvar, Nicola, 1999; } \\
\text { Hirschmann et al., 2008; Benejan } \\
\text { et al., 2014 }\end{array}$ \\
$\begin{array}{l}\text { Macroinvertebrados: redução da diversidade, alteração na composição de espécies, } \\
\text { alteração na dominância de grupos funcionais e alteração na densidade em diferentes } \\
\text { pontos do rio }\end{array}$ & Cortes et al., 1998; Fu et al., 2008 \\
$\begin{array}{l}\text { Zooplâncton: alteração na densidade e na riqueza de espécies em diferentes pontos do } \\
\text { rio }\end{array}$ & Zhou et al., 2009 \\
$\begin{array}{l}\text { Algas: alteração na riqueza de espécies à montante e à jusante, redução da diversidade, } \\
\text { alteração da composição de espécies, redução da porcentagem de indivíduos eretos. }\end{array}$ & Wu et al., 2009; 2010 \\
Menor Índice de Integridade Biológica em locais sob influência de PCHs & Wu et al., 2012 \\
\hline
\end{tabular}


após a construção da PCH (Almodóvar \& Nicola, 1999). Do mesmo modo, as mudanças na estrutura de comunidades foram registradas por meio de modificações de parâmetros demográficos, como abundância relativa e dominância de espécies, e, também, pela alteração da composição de espécies. Uma das pesquisas evidenciou ainda o desaparecimento de nove espécies de peixes após a construção de uma PCH (Hirschmann et al. 2008).

As PCHs, ao alterarem a estrutura de população e de comunidade da ictiofauna, podem acarretar impactos indiretos sobre as atividades de pesca artesanal e afetar o modo de vida dos pescadores e sua relação com o ambiente. Nesse sentido, Hallwass (2011) verificou que a construção da Hidrelétrica de Tucuruí, na Amazônia, ocasionou mudanças na comunidade de peixes, provocando a redução da produção anual e do valor econômico anual da pesca. Hallwass sugeriu que, em função disso, os pescadores podem passar a praticar outras atividades, como agrícolas, pecuárias e madeireiras, para compensar a diminuição da renda com a atividade pesqueira, o que aumentaria os impactos sobre a floresta. Assim, por também alterarem a comunidade ictiofaunística, é possível que as PCHs estejam ocasionando o mesmo tipo de resposta na relação entre pescadores e as populações de peixes, causando prejuízos similares aos das grandes usinas hidrelétricas à sustentabilidade ambiental.

Além da ictiofauna, macroinvertebrados também foram alvos das pesquisas sobre os impactos ambientais de PCHs. Nessas pesquisas, foi constatado que o funcionamento das PCHs afeta a estrutura de comunidades desses organismos, alterando a diversidade de espécies, bem como a distribuição espacial desses organismos ao longo do rio (Cortes et al., 1998; Fu et al., 2008). Os macroinvertebrados exercem um importante papel em ecossistemas lóticos por atuarem ativamente no fluxo de energia e ciclagem de nutrientes (Allan \& Castillo, 2007). Alterações na distribuição e na abundância de uma única espécie podem acarretar respostas compensatórias inesperadas de outras espécies, além de interferir em processos ecológicos, como as taxas de pastejo e decomposição, por exemplo (Covich et al., 1999). Assim, as alterações na comunidade desses organismos têm potencial para causar efeitos secundários em todo o funcionamento do ecossistema e influenciar toda a dinâmica do mesmo.

Algumas pesquisas indicaram também que as PCHs alteram a estrutura de comunidade de algas, afetando parâmetros como riqueza de espécies, diversidade de espécies e porcentagem de grupos morfofuncionais (Wu et al., 2009; 2010). Wu et al. (2012) indicaram também, por meio de um Índice de Integridade Biótica baseado em diatomáceas, que as $\mathrm{PCHs}$ reduzem a qualidade ambiental dos pontos sob sua influência e, portanto, têm impactos negativos sobre esse grupo de organismos.

Além das pesquisas com foco nas alterações bióticas causadas pela implantação de PCHs, outros estudos têm se dedicado a comparar os impactos causados pelas PCHs com os impactos causados por grandes usinas hidrelétricas, considerando a quantidade de energia gerada por cada um dos tipos de empreendimentos. A partir desses estudos, ficou demonstrado que os impactos acumulados por um conjunto de PCHs (por unidade de energia gerada) são similares ou superiores aos impactos provenientes das usinas hidrelétricas de maior porte (Ziv et al., 2012; Bakken et al., 2012; Kliber \& Tullos, 2013).

Além disso, o porte pode não ser o melhor indicador para avaliar os impactos ambientais de plantas de geração de energia elétrica. Gleick (1992) sugere que a relação entre o tamanho da queda bruta e a altura da barragem seria mais adequada para mensurar determinados impactos. Ainda assim, 
observando os dados apresentados por Gleick para a "área inundada", "perda de água por evaporação", "perda de água por infiltração" e "requerimento de água estocada", considerando somente o tamanho (que é o critério usualmente utilizado para classificar os aproveitamentos hidrelétricos), as plantas de geração com menores capacidades instaladas $(<25 \mathrm{MW})$ apresentam impactos maiores do que as plantas de maior capacidade ( $>25 \mathrm{MW}$ ) (Tabela 2 ), o que corrobora os resultados dos outros estudos.

TABELA 2 - Quantificação de impactos gerados por plantas hidrelétricas de acordo com a capacidade instalada (maior que $25 \mathrm{MW}$ - menor que $25 \mathrm{MW}$ ).

\begin{tabular}{|c|c|c|}
\hline Impacto & $>25 \mathrm{MW}$ & $<25 \mathrm{MV}$ \\
\hline $\begin{array}{l}\text { Média de área inundada } \\
\left(\mathrm{km}^{2} / 10^{18} \mathrm{~J}\right)\end{array}$ & 660 & 4000 \\
\hline $\begin{array}{l}\text { Média de perda de água por } \\
\text { evaporação }\left(\mathrm{km}^{3} / 10^{18} \mathrm{~J}\right)\end{array}$ & 0,7 & 4 \\
\hline $\begin{array}{l}\text { Média de requerimento de água } \\
\text { estocada }\left(\mathrm{km}^{3} / 10^{18} \mathrm{~J}\right)\end{array}$ & 6 & 23 \\
\hline $\begin{array}{l}\text { Média de perda por infiltração } \\
\left(\mathrm{km}^{3} / 10^{18} \mathrm{~J}\right)\end{array}$ & 0,3 & 1,1 \\
\hline
\end{tabular}

FONTE: Gleick (1992).

Essas pesquisas apontam para a necessidade de se avaliar os impactos ambientais cumulativos e sinérgicos provenientes da implantação de inúmeras PCHs em uma mesma bacia hidrográfica. No caso da Bacia do Rio Paraíba do Sul, na região Sudeste, por exemplo, está previsto que $117 \mathrm{PCHs}$ deverão entrar em funcionamento até 2025 (AGEVAP, 2012). É necessário que esse conjunto de PCHs seja avaliado e comparado com outras alternativas de fornecimento de energia elétrica, para avaliar se, de fato, são a opção ambientalmente mais adequada.

Essa necessidade de análise aprofundada dos impactos acumulados de um conjunto de PCHs é corroborada pela observação dos resultados apresentados por Mantel et al. (2010) em sua pesquisa. Mantel e seus colaboradores concluem que as alte- rações das características físico-químicas das águas dos rios estão mais correlacionadas com a densidade de pequenas barragens do que com o volume dos reservatórios. Em suma, o número de pequenas barragens instaladas para a construção de várias $\mathrm{PCHs}$ em uma mesma bacia hidrográfica pode ter maior potencial para causar impactos ambientais do que os grandes reservatórios das usinas hidrelétricas, que são distribuídos de forma mais espaçada dentro da paisagem. Este aspecto é salientado por Pang et al. (2015), que sugerem que a implantação de PCHs, de forma cumulativa, pode causar danos comparáveis aos dos empreendimentos de grande porte. Pang e seus colaboradores apontam ainda que os principais impactos causados pelas $\mathrm{PCHs}$ são provenientes da redução de fluxo à jusante da barragem e que, se estes impactos não forem evitados, a história de degradação ambiental causada pelas grandes usinas hidrelétricas pode se repetir com as PCHs.

Além dos impactos sobre o ambiente, a construção de PCHs também causa impactos de cunho socioeconômico na região de implantação dos empreendimentos. Os impactos positivos se materializam pelo aumento da oferta de empregos e aumento da demanda por bens e serviços que acabam por dinamizar as economias locais. Entretanto, é importante mencionar que esses benefícios são mais significativos durante a fase de construção das PCHs e diminuem durante a fase de operação dos empreendimentos (Aguilar, 2006).

A partir da análise dos empregos diretos e indiretos gerados por $59 \mathrm{PCHs}$ integrantes do PROINFA, Tiago e colaboradores (2008) determinaram que as mesmas seriam responsáveis pela criação de 45 mil postos de trabalho durante as fases de construção e operação, o que leva a uma média de 763 empregos para cada PCH. No entanto, Tiago e colaboradores mostram que, durante a fase de operação, uma PCH necessita de apenas 
32 trabalhadores, o que indica que a maioria dos empregos gerados por esse tipo de empreendimento concentra-se nas fases de implantação das PCHs. Portanto, os impactos socioeconômicos positivos mais significativos oriundos das $\mathrm{PCHs}$ são temporários, sendo reduzidos durante a fase de operação das mesmas.

Já em relação aos impactos socioeconômicos negativos, as $\mathrm{PCHs}$ têm como principais consequências impactos semelhantes aos causados por grandes usinas hidrelétricas, porém, em menor escala. Nesse sentido, podem ser citados: aumento da pressão sobre as infraestruturas existentes na região de implantação, aumento de doenças de veiculação hídrica, aumento da probabilidade de contaminação do lençol freático, geração de expectativas na população, a redução de áreas destinadas à agricultura e à pecuária, interferências nas atividades de pesca e interferência no cotidiano das populações (Aguilar, 2006).

Apesar de seu pequeno porte, muitas PCHs ainda necessitam da realocação de famílias, causando conflitos com a população residente na área destinada à sua implantação e a interferência em seus modos de vida (Prado, 2013). Em determinados casos, as PCHs foram instaladas em regiões que interferem em territórios de populações tradicionais, prejudicando o cotidiano dessas populações, bem como a manutenção de suas tradições políticas, sociais e culturais (Grácio, 2009).

Nesse contexto, a bacia do Rio Branco, em Rondônia, pode ser citada como exemplo de região marcada por conflitos socioambientais em função da instalação de diversas PCHs. Os afluentes do Rio Branco tiveram suas vazões totalmente alteradas após a implantação das PCHs, interferindo nas atividades de pequenos agricultores e populações indígenas, que tentam conseguir algum tipo de compensação pelos prejuízos sofridos há vários anos por meio do judiciário (Sevá Filho et al., 2011)

\section{A fragilização dos instrumentos de controle ambiental em virtude das facilitações concedidas às $\mathrm{PCH}$}

Como apontado nas seções anteriores, diversos incentivos econômicos foram criados para aumentar a atratividade da construção de $\mathrm{PCHs}$ por investidores privados. De forma concomitante, a aprovação desses empreendimentos tem sido facilitada a partir da flexibilização da legislação que regula o processo de licenciamento ambiental. A combinação dessas estratégias tem potencial para fragilizar os instrumentos de controle ambiental existentes no Brasil. Em adição, também podem contribuir para reforçar a percepção de que o processo de licenciamento ambiental é um obstáculo para o desenvolvimento econômico e precisa ser contornado (Zhouri \& Laschefski, 2010).

A fragilização do processo de licenciamento ambiental é exemplificada pela possibilidade de substituição do EIA (que é um componente central no processo de AIA) por um RAS no licenciamento das PCHs. Esta substituição enfraquece o processo de avaliação de impactos ao se substituir um estudo completo e aprofundado por outro que possui um escopo reduzido. O fato é que o RAS não contempla a análise dos impactos cumulativos e sinérgicos e os especialistas que o produzem não são obrigados a considerar a totalidade de uma bacia hidrográfica para definir a área de influência do projeto, pois estes não são requisitos presentes na Resolução CONAMA 279/2001, que trata do assunto. Tais requisitos são fundamentais para o contexto brasileiro, onde diversas PCHs são construídas numa mesma bacia hidrográfica. Portanto, a substituição do EIA pelo RAS pode impedir que o processo de licenciamento e a AIA cumpram seu objetivo básico, que é o de evitar ou minimizar a ocorrência de 
impactos ambientais em função do estabelecimento de determinados empreendimentos.

Outra estratégia utilizada para contornar os supostos entraves para o desenvolvimento econômico foi a redução no tempo disponível para que o órgão ambiental analise os estudos realizados pelo empreendedor e emita a licença ambiental que está sendo requerida. Essa redução é outro aspecto que possui forte potencial para comprometer a qualidade do processo de licenciamento ambiental e a AIA. É que, mesmo com mais tempo para analisar os estudos ambientais realizados pelos empreendedores, os órgãos ambientais têm aprovado estudos de má qualidade e com informações incompletas. Um caso exemplar dessa situação é o da Usina Hidrelétrica de Belo Monte, que teve diversas deficiências apontadas em seu EIA/RIMA por um grupo de especialistas independentes (Bermann, 2012). Com a redução no tempo de análise, é plausível assumir que os estudos realizados pelos empreendedores sejam avaliados de forma menos rigorosa e que novos casos como o de Belo Monte se repitam, acarretando assim um acúmulo de prejuízos sociais e ambientais pelo país.

As flexibilizações realizadas no processo de aprovação das $\mathrm{PCHs}$ são uma consequência da lógica subjacente à prática do licenciamento ambiental vigente no país. De acordo com Zhouri (2012), o licenciamento ambiental brasileiro é baseado no Paradigma da Adequação Ambiental, onde algumas externalidades socioambientais são incluídas no projeto, contudo sem torná-lo inviável do ponto de vista econômico. Segundo Zhouri, desse modo o que prevalece é o uso dos recursos naturais a partir dos pressupostos do mercado, onde os impactos sociais e ambientais são reduzidos a barreiras a serem transpostas em nome do "interesse público" ou do "interesse nacional". Nesse contexto de eliminação de barreiras, os impactos de determinado projeto são circunscritos apenas a aspectos mitigáveis e compensáveis, o que limita as contribuições do licenciamento e da AIA para a concepção de projetos mais sustentáveis e para a incorporação dos aspectos socioambientais no processo decisório (Montaño, 2014).

Assim, o que deveremos ter será a manutenção da supremacia da racionalidade econômica em detrimento da sustentabilidade socioambiental, nos projetos de desenvolvimento econômico em curso no Brasil. Essa supremacia acarretará a fragilização dos processos de licenciamento e da AIA, instrumentos ambientais instituídos pela Política Nacional de Meio Ambiente e ratificados na Constituição Federal de 1988, para atender às necessidades dos agentes do mercado. Nesse sentido, Zhouri et al. (2005) enfatizam que já tem ocorrido uma reinterpretação das leis que tratam de matéria ambiental no Brasil para adequar as mesmas a projetos econômicos. De forma adicional, Capra (2002) sugere que essa desregulamentação pode ser interpretada como uma consequência da busca pelo crescimento dentro de uma economia globalizada, onde a legislação ambiental no âmbito dos Estados Nacionais é enfraquecida com o objetivo de baixar custos e aumentar a competitividade em escala global.

O predomínio da racionalidade econômica no modelo de desenvolvimento adotado pelo Brasil mostra o descompasso do mesmo com os princípios da constituição e da legislação ambiental vigente, o que, muitas vezes, fere diretamente o "princípio do não retrocesso ecológico" (Vilani, 2013). Este princípio deveria ser observado para garantir que novas normas não alterem as anteriores por meio de dispositivos menos restritivos, com potencial para permitir maior grau de degradação ou poluição ambiental (Prieur, 2011). Entretanto, um movimento oposto ao não retrocesso é o que efetivamente tem sido observado. 
A Resolução CONAMA 279/2001, que flexibiliza o licenciamento ambiental das PCHs, constitui um exemplo, entre muitos outros, de violação do princípio do não retrocesso ecológico (Schaedler \& Winkler, 2013). Esse caso é especialmente preocupante, pois trata de um tipo de empreendimento que vem sendo amplamente construído no Brasil, que, no entanto, não tem suas consequências socioambientais conhecidas com rigor e suporte científico suficiente para validar sua sustentabilidade. A pesquisa sobre os impactos ambientais de PCHs ainda não constitui um campo de conhecimento consolidado, ao contrário das grandes usinas hidrelétricas. Em virtude disso, o lógico seria, ao invés de retroceder ambientalmente, se basear no Princípio da Precaução no processo de aprovação de PCHs, o qual deve ser utilizado sempre que as probabilidades de ocorrência de danos ambientais não forem bem conhecidas (Setzer \& Gouveia, 2010).

Desse modo, um aspecto que precisa ser enfatizado é que, dados os elementos já conhecidos sobre seus efeitos ambientais, as PCHs deveriam ser submetidas a um amplo processo de avaliação de impactos e licenciamento ambiental, sendo observadas as etapas e normas estabelecidas pela Política Nacional de Meio Ambiente e pelas resoluções CONAMA 001/1986 e 237/1997, além das boas práticas estabelecidas internacionalmente. As lacunas utilizadas para agilizar o processo de licenciamento ambiental de PCHs se baseiam na hegemonia da racionalidade econômica e, portanto, não garantem que a dimensão socioambiental seja levada em consideração nos processos decisórios. Como consequência, tais lacunas impedem que a AIA e o licenciamento ambiental cumpram seu objetivo básico de proteger o ambiente e ainda ratificam a perspectiva reducionista de entrave burocrático que tem sido atribuída a esses instrumentos.

\section{Conclusões}

O Brasil tem construído um cenário propício para o investimento em PCHs visando aproveitar os pequenos potenciais hidrelétricos ainda existentes nas regiões não amazônicas do país. Tal cenário tem sido materializado por meio da concessão de incentivos fiscais, financeiros e comerciais e de programas governamentais que favorecem a implantação desse tipo de empreendimento. Além disso, o governo federal tem apostado na flexibilização das normas ambientais relativas ao licenciamento de empreendimentos elétricos com baixo potencial de causar impactos, com o objetivo de agilizar a aprovação e a implantação desses empreendimentos.

Esses incentivos e flexibilizações têm sido concretizados com base no pressuposto de que as PCHs causam baixos impactos ambientais e, portanto, produzem um tipo de energia "limpa". Entretanto, a revisão que realizamos da literatura científica que trata desse tipo de empreendimento demonstra que as PCHs, apesar da baixa capacidade instalada e do menor tamanho de suas barragens, são capazes de afetar as características hidrológicas dos ecossistemas aquáticos e biota em nível individual, populacional e de comunidades, bem como causar impactos negativos às populações que vivem nas regiões onde esses empreendimentos são implantados. Além disso, quando os impactos de várias PCHs são analisados de forma cumulativa e são comparados com os impactos de grandes usinas hidrelétricas, foi constatada uma tendência de que o conjunto de PCHs apresente impactos tão ou mais significativos do que aqueles causados pela construção e funcionamento de grandes usinas hidrelétricas, especificamente quando a quantidade de energia gerada é levada em consideração. 
Diante desses resultados, as flexibilizações e incentivos dados à implantação de PCHs no Brasil precisam ser colocados em questão. Não existem evidências científicas suficientes para validar a premissa de sustentabilidade desses empreendimentos, principalmente quando sua implantação ocorre de forma adensada em uma mesma bacia hidrográfica. Portanto, não há justificativa para facilitar a disseminação desse tipo de planta geradora de energia pelo país e, menos ainda, justificativa para afrouxar as restrições ambientais impostas a elas.

Com base nas evidências aqui elencadas, ficou demonstrado que as PCHs devem passar por uma avaliação ambiental criteriosa, como qualquer outro empreendimento potencialmente poluidor, de acordo com o preconizado pela Política Nacional de Meio Ambiente. Além disso, tais avaliações devem ser realizadas com maior rigor, já que o conhecimento sobre os impactos ambientais de PCHs ainda é relativamente escasso. Nesses casos, o mais adequado seria recorrer ao princípio da precaução no processo de aprovação de PCHs e não permitir o retrocesso ecológico por meio de normas menos restritivas.

\section{Referências}

Abassi, S. A.; Abassi, N. The likely adverse environmental impacts of renewable energy sources. Applied Energy, 65, 121-144, 2000. doi: 10.1016/S0306-2619(99)00077-X

Abassi, T.; Abassi, S. A. Small hydro and the environmental implications of its extensive utilization. Renewable and Sustainable Energy Reviews, 15, 2134-2143, 2011. doi: 10.1016/j.rser.2010.11.050

AGEVAP - Associação Pró-Gestão das Águas da Bacia Hidrográfica do Rio Paraíba do Sul. Estudos com objetivo de avaliar os impactos de novas transposições de vazão no Rio Paraíba do Sul. R2: Levantamento de dados. AGEVAP, 2012. Disponível em: <http://ceivap.org.br/conteudo/r2-levantamento-de-dados-final.pdf $>$.
A racionalidade econômica não deve ser suficiente para facilitar e agilizar os processos de aprovação e implementação de PCHs. Assim sendo, é importante notar que as estratégias utilizadas para tornar o processo de aprovação de uma $\mathrm{PCH}$ mais expedito terminam por fragilizar os processos de AIA e licenciamento ambiental e contribuem para que tais instrumentos continuem a ser vistos como entraves ao desenvolvimento econômico. Em última instância, a fragilização do processo de licenciamento ambiental contribuirá para manter as preocupações com a preservação ambiental em patamares inferiores nas decisões tomadas no setor elétrico brasileiro, fazendo com que danos sociais e ambientais se acumulem por todo o país.

Finalmente, o presente artigo não sugere que as PCHs deixem de fazer parte das estratégias para expansão da oferta de energia elétrica do Brasil. Entretanto, a tese aqui levantada é de que se abandone a percepção dominante de que as PCHs possuem impactos ambientais insignificantes e que se adote um procedimento de avaliação de impactos sociais e ambientais baseado nas melhores práticas e normas existentes.

Aguilar, G. T. Avaliação de Impacto Social e proposição de medidas mitigadoras - compromisso com a Responsabilidade Social. PCH Noticias e SHP News, 31, 12-17, 2006. Disponível em: $<$ http://cerpch.unifei.edu.br/artigos/ avaliacao-de-impacto-social-e-proposicao-de-medidas-mitigadoras-compromisso-com-a-responsabilidade-social/ $>$.

Albuquerque, R. M.; Moraes, G. G. Eletroestratégias: as pequenas centrais hidrelétricas e os meandros do setor elétrico brasileiro. Campo-Território: Revista de Geografia Agrária, 8(16), 379-398, 2013. doi: 10.1002/(SICI)1099-1646(199909/10)15:5<477::AID-RRR560>3.0.CO;2-B

Allan, J. D.; Castillo, M. M. Stream Ecology. Structure and function on running waters. 2. ed. Springer, 2007. 
Almodóvar, A.; Nicola, G. G. Effects of a small hydropower station upon brown trout Salmo trutta L. in the river Hoz Seca (Tagus Basin, Spain) one year after regulation. Regulated Rivers: Research Management, 15, 477-484, 1999. doi: 10.1002/(SICI)1099-1646(199909/10)15:5<477::AIDRRR560>3.0.CO;2-B

ANEEL - Agência Nacional de Energia Elétrica. Resolução 394, de 04 de dezembro de 1998. Estabelece os critérios para o enquadramento de empreendimentos hidrelétricos na condição de pequenas centrais hidrelétricas. Brasília: DOU de 07/12/1998.

ANEEL - Agência Nacional de Energia Elétrica. Resolução 652, de 09 de dezembro de 2003. Estabelece os critérios para o enquadramento de aproveitamento hidrelétrico na condição de Pequena Central Hidrelétrica (PCH). Brasília: DOU de 10/12/2003a.

ANEEL - Agência Nacional de Energia Elétrica. Guia do empreendedor de pequenas centrais hidrelétricas. Brasília, DF, ANEEL, 2003b.

Bakken, T. H.; Sundt, H.; Ruud, A.; Harby, A. Development of small versus large hydropower in Norway comparison of environmental impacts. Energy Procedia, 20, 185-199, 2012. doi: 10.1016/j.egypro.2012.03.019.

Barbosa, A. C.; Souza, M. M. Incentivos às pequenas centrais hidrelétricas (PCHs): PROINFA e MDL. 2009. Disponível em: <http://www.renenergyobservatory.org/ br.html>.

Baxter, R. M. Environmental effects of dams and impoundments. Annual Review of Ecology, Evolution, and Systematics, 8, 255-283, 1977. doi: 10.1146/annurev. es.08.110177.001351

Benejan, L.; Saura-Mas, S.; Bardina, M.; Solà, C.; Munné, A.; García-Berthou, E. Ecological impacts of small hydropower plants on headwater stream fish: from individual to community effects. Ecology of Freshwater Fish, 2014. doi: 10.1111/eff.12210.

Bermann, C. Impasses e controvérsias da hidreletricidade. Estudos Avançados, 21(59), 139-153, 2007. doi: 10.1590/ S0103-40142007000100011.

Bermann, C. O projeto da usina hidrelétrica de Belo Monte: a autocracia energética como paradigma. Novos Cadernos NAEA, 15(1), 5-23, 2012. doi: 10.5801/ncn.v15i1.895
Brasil. Lei 10.438, de 26 de abril de 2002. Dispõe sobre a expansão da oferta de energia elétrica emergencial, recomposição tarifária extraordinária, cria o Programa de Incentivo às Fontes Alternativas de Energia Elétrica (PROINFA), a Conta de Desenvolvimento Energético (CDE), dispõe sobre a universalização do serviço público de energia elétrica, dá nova redação às Leis n. 9.427, de 26 de dezembro de 1996, n. 9.648, de 27 de maio de 1998, n. 3.890-A, de 25 de abril de 1961, n. 5.655, de 20 de maio de 1971, n. 5.899, de 5 de julho de 1973, n. 9.991, de 24 de julho de 2000, e dá outras providências. Brasília: DOU de 29/04/2002.

Brasil. Decreto 5.025, de 30 de março de 2004. Regulamenta o inciso I e os $\S \S 1^{\circ}, 2^{\circ}, 3^{\circ}, 4^{\circ}$ e $5^{\circ}$ do art. $3^{\circ}$ da Lei n. 10.438 , de 26 de abril de 2002, no que dispõem sobre o Programa de Incentivo às Fontes Alternativas de Energia Elétrica - PROINFA, primeira etapa, e dá outras providências. Brasília: DOU de 31/03/2004.

Capra, F. As conexões ocultas. Ciência para uma vida sustentável. São Paulo: Cultrix, 2002.

Castro, N. J.; Martini, S.; Brandão, R.; Dantas, G. A.; Tiponi, R. R. A importância das fontes alternativas e renováveis na evolução da matriz elétrica brasileira. In: V Seminário de Geração e Desenvolvimento Sustentável, Fundación MAPFRE. São Paulo, 25 de agosto de 2009.

CONAMA - Conselho Nacional de Meio Ambiente. Resolução 001, de 23 de janeiro de 1986. Dispõe sobre critérios básicos e diretrizes gerais para a avaliação de impacto ambiental. Brasília: DOU de 17/02/1986.

CONAMA - Conselho Nacional de Meio Ambiente. Resolução 237, de 19 de dezembro de 1997. Dispõe sobre a revisão e complementação dos procedimentos e critérios utilizados para o licenciamento ambiental. Brasília: DOU de 22/12/1997.

CONAMA - Conselho Nacional de Meio Ambiente. Resolução 279, de 27 de junho de 2001. Brasília: DOU de 19/06/2001.

Cortes, R. M. V.; Ferreira, M. T.; Oliveira, S. V.; Godinho, F. Contrasting impact of small dams on the macroinvertebrates of two Iberian mountain rivers. Hydrobiologia 389, 51-61, 1998. doi: 10.1023/A:1003599010415

Covich, A. P.; Palmer, M. A.; Crowl, T. A. The role of benthic invertebrate species in freshwater ecosystems. Zoobenthic species influence energy flows and nutrient cycling. BioScience, 49(2), 119-127, 1999. doi: 10.2307/1313537 
DNAEE - Departamento Nacional de Águas e Energia Elétrica. Portaria n. 109, de 24 de novembro de 1982. Brasília: DOU de 26/11/1982.

DNAEE - Departamento Nacional de Águas e Energia Elétrica. Portaria n. 136, de 06 de outubro de 1987. Brasília: DOU de 08/10/1987.

ELETROBRÁS - Centrais Elétricas Brasileiras. Diretrizes para estudos e projetos de Pequenas Centrais Hidrelétricas. Brasília: Eletrobras, 2000.

EPE - Empresa de Pesquisa Energética. Plano Decenal de Expansão de Energia. 2008-2017. Rio de Janeiro: EPE, 2009.

EPE - Empresa de Pesquisa Energética. Plano Decenal de Expansão de Energia 2023. Rio de Janeiro: EPE, 2014.

Fu, X.; Tang, T.; Jiang, W.; Li, F.; Wu, N.; Zhou, S.; Cai, Q. Impacts of small hydropower plants on macroinvertebrate communities. Acta Ecologica Sinica, 28(1), 45-52, 2008. doi: 10.1016/S1872-2032(08)60019-0

Gleick, P. H. Environmental consequences of hydroelectric development: the role of facility size and type. Energy, 17(8), 735-747, 1992. doi: 10.1016/0360-5442(92)90116-H

Grácio, H. G. Notas preliminares sobre os impactos ambientais e sociais gerados pela implantação da pequena central hidrelétrica - PCH Paratinga II em terras tradicionalmente ocupadas pelo povo Xavante. Revista Estudos Amazônicos: Fronteiras e Territórios, 1(1), 97-122, 2009. Disponível em: $<$ http://revista.uft.edu.br/index.php/amazonidas/article/ view/20/32>.

Hallwass, G. Ecologia humana da pesca e mudanças ambientais no Baixo Rio Tocantins, Amazônia Brasileira. Porto Alegre, Dissertação (Mestrado em Ecologia) - UFRGS, 2011.

Hirschmann, A.; Majolo, M. A.; Grillo, H. C. Z. Alterações na ictiocenose do rio Forqueta em função da instalação da Pequena Central Hidrelétrica Salto Forqueta, Putinga, Rio Grande do Sul. Iheringia, Série Zoologia, 98(4), 481-488, 2008. doi: 10.1590/S0073-47212008000400011

IPCC - Intergovernmental Panel on Climate Change. Renewable Energy Sources and Climate Change Mitigation. Cambridge: Cambridge University Press, 2012.

Kahn, J. R.; Freitas, C. E.; Petrere, M. False shades of green: the case of Brazilian Amazonian Hydropower. Energies, 7, 6063-6082. 2014. doi: 10.3390/en7096063
Kliber, K. M.; Tullos, D. D. Cumulative biophysical impact of small and large hydropower development in Nu River, China. Water Resource Research, 49, 1-15, 2013. doi: 10.1002/wrcr.20243

Leão, L. L.; Brasil Junior, A. C. P. Mecanismos de incentivo à construção de pequenas centrais hidrelétricas. In: Anais do IV Encontro da Associação Nacional de Pesquisa e Pós-Graduação em Ambiente e Sociedade. Brasília, Distrito Federal, 4-6 de jun. 2008.

Lopes, L. V. Política energética e fontes alternativas no Brasil. Revista Gestão e Conexões, 4(2), 144-163, 2015. doi: 10.13071/regec.2317-5087.2015.4.2.6542.144-163

Mantel, S. K.; Hughes, D. A.; Muller, M. W. K. Ecological impacts of small dams on South African rivers Part 1: Drivers of change - water quantity and quality. Water $S A, 36(3)$, 351-360, 2010. Disponível em: <http://www.wrc.org.za>

Marrocos Neto, A. A. S.; Moret, A. S. Contribuição técnica, econômica e ambiental das PCHs no Sistema Elétrico Isolado de Rondônia. Espaço Energia, 9, 25-33, 2008. Disponível em: <http://www.espacoenergia.com.br/edicoes/9/ EE009-05.pdf $>$.

Mello, F. M.; Piasetin, C. A história das barragens no Brasil, Séculos XIX, XX e XXI: cinquenta anos do Comitê Brasileiro de Barragens. Rio de Janeiro: CBDB - Comitê Brasileiro de Barragens, 2011.

Montaño, M. Planejamento às avessas: os descompassos da avaliação de impactos sociais no Brasil. In: Oliveira, J. P; Cohn, C. (Org.). Belo Monte e a questão indígena. Brasília: ABA Publicações, 2014. p. 33-49.

Moretto, E. M.; Gomes, C. S.; Roquetti, D. R.; Jordão, C. O. Histórico, tendências e perspectivas no planejamento espacial de usinas hidrelétricas: a antiga e a atual fronteira amazônica. Ambiente e Sociedade, 15(3), 141-164, 2012. doi: 10.1590/S1414-753X2012000300009

Pang, M.; Zhang, L.; Ulgiati, S.; Wang, C. Ecological impacts of small hydropower in China: Insights from an energy analysis of a case plant. Energy Policy, 76, 112122, 2015. doi:10.1016/j.enpol.2014.10.009

Poff.; N. L.; Allan, J. D.; Bain, M. B.; Karr, J. R.; Prestegaard, K. L.; Richter, B. D.; Sparks, R. E.; Stromberg, J. C. The natural flow regime. A paradigm for river conservation and restoration. BioScience, 47(11), 769-784, 1997. doi: $10.2307 / 1313099$ 
Prado, R. J. Breve reflexão socioambiental sobre PCHs na bacia do rio Banco em Alta Floresta do Oeste - RO. Revista Brasileira de Ciências da Amazônia, 2(1), 11-21, 2013. Disponível em: $<$ https://sites.google.com/site/simposiohidricodazonadamata/trabalhos $>$.

Premalatha, M.; Tabassum-Abassi; Tasneem-Abassi; Abassi, S. A. A critical view on the eco-friendliness of small hydroelectric installations. Science of the Total Environment, 481, 638-643, 2014. doi: 10.1016/j.scitotenv.2013.11.047

Prieur, M. O princípio da proibição de retrocesso ambiental. In: Comissão de Meio Ambiente, Defesa do Consumidor e Fiscalização e Controle (CMA). Princípio da proibição de retrocesso ambiental. Brasília: Senado Federal, 2011. p. 11-54. Disponível em: <http://www2.senado.leg.br/bdsf/ item/id/242559>.

Ricklefs, R. E. A economia da natureza. 6. ed. Rio de Janeiro: Guanabara Koogan, 2010.

Schaedler, P. F.; Winkler, S. T. Retrocessos no regime de proteção ambiental do licenciamento de usinas hidroelétricas: um caso de desobediência à Constituição Federal de 1988. Revista Jurídica CCJ, 17(33), 21-48, 2013. Disponível em: $<$ http://proxy.furb.br/ojs/index.php/juridica/article/ viewFile/2422/2371>.

Setzer, J; Gouveia, N. Princípio da Precaução: da origem ética à sua aplicação prática. In: Ribeiro, W. C. (Org.). Rumo ao pensamento crítico socioambiental. São Paulo: Annablume, 2010. p. 35-53.

Sevá Filho, A. O.; Nobrega, R. S.; Garzon, L. F. N. Rios de Rondônia: jazidas de megawatts e expropriação social e ambiental. In: V Encontro Nacional da ANPUR, Rio de Janeiro, 23-27 de maio, 2011.

Souza, P. A. P.; Felicidade, N.; Mauad, F. F. Planejamento e gestão ambiental integrada quando da implantação de pequenas centrais hidrelétricas (PCHs). In: Anais do II Simpósio de Recursos Hídricos do Centro Oeste, Campo Grande, 23-26 de jun. 2002.

Tiago, G. L. Brasil redescobre potencial das micro e pequenas centrais hidrelétricas (PCHs). In: Greenpeace. Energia Positiva para o Brasil. Greenpeace, 2004.

Tiago, G. L.; Galhardo, C. R.; Antloga, J. G.; Ferrari, J. T. Um panorama das pequenas centrais no Brasil. Revista PCH Noticias e SHP News, 33, 19-23, 2011. Disponível em: <http://www.cerpch.unifei.edu.br/arquivos/artigos/ pch-shp-33-pag19a23.pdf>.

Tiago, G. L.; Galhardo, C. R.; Duarte, E. R. B. C.; Nascimento, J. G. A. Impactos socioeconômicos das pequenas centrais hidrelétricas inseridas no Programa de Incentivo às Fontes Alternativas de Energia (PROINFA). Revista Brasileira de Energia, 14(1), 145-166, 2008. Disponível em: $<$ http://new.sbpe.org.br/artigo/impactos-socio-economicos-das-pequenas-centrais-hidreletricas-inseridas-no-programa-de-incentivo-fontes-alternativas-de-energia-proinfa/ $>$.

Vilani, R. M. Legislação e política ambiental no Brasil: as possibilidades de desenvolvimento sustentável e os riscos do retrocesso ambiental. Revista Brasileira de Pós-Graduação, 10(21), 829-860, 2013. Disponível em: <http://ojs. rbpg.capes.gov.br/index.php/rbpg/article/view/414/345>.

WCD - World Commission on Dams. Dams and development. A new framework for decision making. Earthscan Publications, 2000.

Wu, N.; Tang, T.; Zhou, S.; Jia, X.; Li, D.; Liu, R.; Cai, Q. Changes in benthic algal communities following construction of a run-of-river dam. Journal of the North American Benthological Society, 28(1), 69-79, 2009. doi: 10.1899/08-047.1

Wu, N.; Tang, T., Fu, X.; Jiang, W.; Li, F.; Zhou, S. Cai, Q.; Foher, N. Impacts of cascade run-of-river dams on benthic diatoms in the Xiangxi River, China. Aquatic Sciences, 72, 117-125, 2010. doi: 10.1007/s00027-009-0121-3

Wu, N.; Cai, Q.; Fohrer, N. Development and evaluation of a diatom-based index of biotic integrity (D-IBI) for rivers impacted by run-of-river dams. Ecological Indicators, 18, 108-117, 2012. doi: 10.1016/j.ecolind.2011.10.013

Zhou, S.; Tang, T.; Wu, N.; Fu, X.; Jiang, W.; Li, F.; Cai, Q. Impacts of cascaded small hydropower plants on microzooplankton in Xiangxi River, China. Acta Ecologica Sinica, 29, 62-68, 2009. doi: 10.1016/j.chnaes.2009.04.008

Zhouri, A. Belo Monte: crise do sistema ambiental e da democracia. In: Zhouri, A. (Org.). Desenvolvimento, reconhecimento de direitos e conflitos territoriais. Brasília: ABA, 2012. p. 45-65.

Zhouri, A.; Laschefski, K. Desenvolvimento e conflitos ambientais: um novo campo de investigação. In: Zhouri, A. Laschefski, K. (Orgs.). Desenvolvimento e conflitos ambientais. Belo Horizonte: Editora UFMG, 2010. p. 11-33. 
Zhouri, A.; Laschefski, K.; Paiva, A. Uma sociologia do licenciamento ambiental: o caso das hidrelétricas em minas gerais. In: Zhouri, A.; Perreira, D. B.; Laschefski, K. (Orgs.). A insustentável leveza da política ambiental: desenvolvimento e conflitos socioambientais. 1. ed. Belo Horizonte: Autêntica Editora, 2005. p. 89-118.
Ziv, G.; Baran, E.; Nam, S.; Rodríguez-Iturbe, I.; Levin, S. A. Trading-off fish biodiversity, food security, and hydropower in the Mekong River Basin. PNAS, 109(15), 5609-5614, 2012. doi: 10.1073/pnas.1201423109 